\title{
Flow cytometric detection of hyper-polarized mitochondria in regulated and accidental cell death processes
}

\author{
G. Warnes ${ }^{1}$ (D) \\ Published online: 3 June 2020 \\ (c) The Author(s) 2020
}

\begin{abstract}
Shikonin induced necroptosis in Jurkat cells were identified flow cytometrically by the up-regulation of RIP3 in live cells and that a proportion of these cells underwent other forms of regulated cell death (RCD) which included parthanatos $(<10 \%)$, or cleaved PARP $(<10 \%)$ and DNA Damage $(>30 \%)$. Live necroptotic cells also possessed functioning mitochondria with hyper-polarized mitochondria membrane potential and generated a fivefold increase in cellular reactive oxygen species (ROS) which was resistant to inhibition by zVAD and necrostatin-1 (Nec-1). After loss of plasma membrane integrity these dead necroptotic cells then showed a higher incidence of parthanatos ( $>40 \%)$, or cleaved PARP $(>15 \%)$ but less DNA Damage $(<15 \%)$. Inhibition of shikonin induced apoptosis and necroptosis by zVAD and Nec-1 respectively resulted in live necroptotic cells with an increased incidence of cleaved PARP and reduced levels of DNA Damage respectively. Dead necroptotic cells then showed a reduced incidence of parthanatos and DNA Damage after inhibition by zVAD and Nec-1 respectively. A high proportion of these dead necroptotic cells (30\%) which lacked plasma membrane integrity also displayed functioning hyper-polarized mitochondria with high levels of cellular ROS and thus had the capacity to influence the outcome of RCD processes rather than just been the end product of cell death, the necrotic cell. Flow cytometry can thus measure multiple forms of RCD and the level of cellular ROS and MMP which highlights the inter-connection between cell death processes and that a single cell may simultaneously display multiple forms of RCD.
\end{abstract}

Keywords Mitochondrial function $\cdot \operatorname{ROS} \cdot \mathrm{RCD} \cdot \mathrm{ACD} \cdot$ Parthanatos $\cdot$ DNA damage

\begin{tabular}{|c|c|c|}
\hline Abbreviations & & LAPO \\
\hline $\mathrm{ACD}$ & Accidental cell death & MFI \\
\hline ATP & Adenosine triphosphate & MLKL \\
\hline CamK II & $\mathrm{Ca} 2+/$ Calmodulin-dependent kinase II & \\
\hline Cleaved PARP & Poly (ADP-ribose) polymerase 1 & MMP \\
\hline DDR & DNA damage response & MPT \\
\hline $\mathrm{DN}$ & Double negative & \\
\hline DNA DSBs & double strand DNA breaks & $\mathrm{NAD}+$ \\
\hline Drp1 & Dynamin guanosine tri-phosphatase & Nec-1 \\
\hline EAPO & Early apoptosis & PDC \\
\hline$\gamma \mathrm{HA} 2 \mathrm{X}$ & Histone A2 family $\mathrm{X}$ & PGAM5 \\
\hline $\begin{array}{l}\text { Electronic supplen } \\
\text { article (https://doi } \\
\text { supplementary ma }\end{array}$ & $\begin{array}{l}\text { entary material The online version of this } \\
\text { rg/10.1007/s } 10495-020-01613-5 \text { ) contains } \\
\text { erial, which is available to authorized users. }\end{array}$ & $\begin{array}{l}\text { RCD } \\
\text { RIP1 }\end{array}$ \\
\hline $\begin{array}{l}\triangle \text { G. Warnes } \\
\text { g.warnes@qm }\end{array}$ & 1.ac.uk & $\begin{array}{l}\text { RIP1 APO } \\
\text { RIP3 }\end{array}$ \\
\hline $\begin{array}{l}1 \text { Flow Cytome } \\
\text { and The Lond } \\
\text { Mary London } \\
\text { UK }\end{array}$ & $\begin{array}{l}\text { y Core Facility, The Blizard Institute, Barts } \\
\text { n School of Medicine and Dentistry, Queen } \\
\text { Jniversity, } 4 \text { Newark Street, London E1 2AT, }\end{array}$ & $\begin{array}{l}\text { ROS } \\
\text { RT }\end{array}$ \\
\hline
\end{tabular}

Late apoptosis

Median fluorescence intensity

Pseudokinase mixed lineage kinase

domain-like protein

Mitochondrial membrane potential

Mitochondrial permeability transition

pore complex

Nicotinamide adenine dinucleotide

Necrostatin-1

Pyruvate dehydrogenase complex

PGAM Family Member 5, Mitochondrial Serine/Threonine Protein Phosphatase

Quadruple negative

Regulated cell death

Receptor-interacting serine/threonineprotein kinase 1

RIP1-dependent apoptosis

Receptor-interacting serine/threonineprotein kinase 3

Reactive oxygen species

Room temperature 
TNF $\alpha \quad$ Tumour Necrosis Factor alpha

TRADD TNF receptor type 1-associated with death domain protein

TRAF-2 TNFR-associated factor 2 forming complex I

zVAD Carbobenzoxy-valyl-alanyl-aspartyl-[Omethyl]- fluoromethylketone

\section{Introduction}

The regulated necrotic cell death process (RCD) necroptosis is a caspase independent process which after TNF $\alpha$ binding to the TNF receptor becomes associated with TRADD (TNF receptor type 1-associated with death domain protein) and TRAF-2 (TNFR-associated factor 2 forming complex I [1-6]). This complex together with the RIP1 protein then auto-phosphorylates after inhibition of caspase-8 (active caspase- 8 cleaves RIP1), [7] which then acts as the first ROS sensor in this form of regulated cell death $[2-6,8]$. This ultimately leads to the up-regulation and phosphorylation of RIP3 forming the so called necrosome and to the recruitment and phosphorylation of MLKL (pseudokinase mixed lineage kinase domain-like protein) at the plasma membrane leading to cell lysis Fig. 1 [5, 6, 9-12]. RIP3 has also been reported to move to the inner mitochondrial membrane where it's interaction with PDC, CamK II, Drp1 and PGAM5 leads to the enhanced production of ROS by mitochondria Fig. 1 [3, $13,14]$. ROS generated during necroptosis has been previously shown to enhance auto-phosphorylation of RIP1 and was essential for RIP3 recruitment to the necrosome leading to enhanced formation of functional necrosomes, with Reactive Oxygen Species (ROS) acting in a positive feedback loop ensuring maintenance of necroptosis (Fig. 1). RIP1 is mainly located in the cytoplasm in the form of the necrosome as part of the necroptosis process, however nuclear RIP1 has also been shown to associate in a kinase independent manner and activate PARP1 inducing PARP1-dependent regulated necrosis or parthanatos Fig. 1 [15].

The drug shikonin is a naturally occurring naphthoquinone has like TNF $\alpha[4,16]$ been reported to induce apoptosis and necroptosis, as well as generate ROS (Fig. 1 [3, 8, 17, 18]. Necrostatin-1 (Nec-1) has been reported to inhibit necroptosis and also the generation of ROS by mitochondria but to a lesser extent in the cytoplasm Fig. $1[3,8,16]$. Shikonin has also been reported to increase the level of $\gamma \mathrm{H} 2 \mathrm{AX}$ or DNA Damage (as part of the DNA Damage Response, DDR), cleaved PARP and also the hyper-activation of
Fig. 1 Shikonin induction of necroptosis, apoptosis, DNA Damage, cleaved PARP and parthanatos. (1) Shikonin is taken up by the cell which causes (2) RIP1 auto-phosphorylates which phosphorylates RIP3 to form the necrosome which can be blocked by Nec-1. (3) The necrosome then phosphorylates MLKL leading to the permeablisation of the plasma membrane and cell death by necroptosis. (4) Phosphorylated RIP proteins move to the mitochondria leading to raised MMP or hyperpolarization of mitochondria and excessive production of ROS. (5) ROS causes DNA Damage leading to phosphorylation of histone $\gamma \mathrm{H} 2 \mathrm{AX}$ which can be blocked by Nec-1. (6) PARP is cleaved by active caspase- 3 which is generated during apoptosis (7). Parthanatos occurs when phosphorylated $\gamma \mathrm{H} 2 \mathrm{AX}$ hyper-activates cleaved PARP (8)

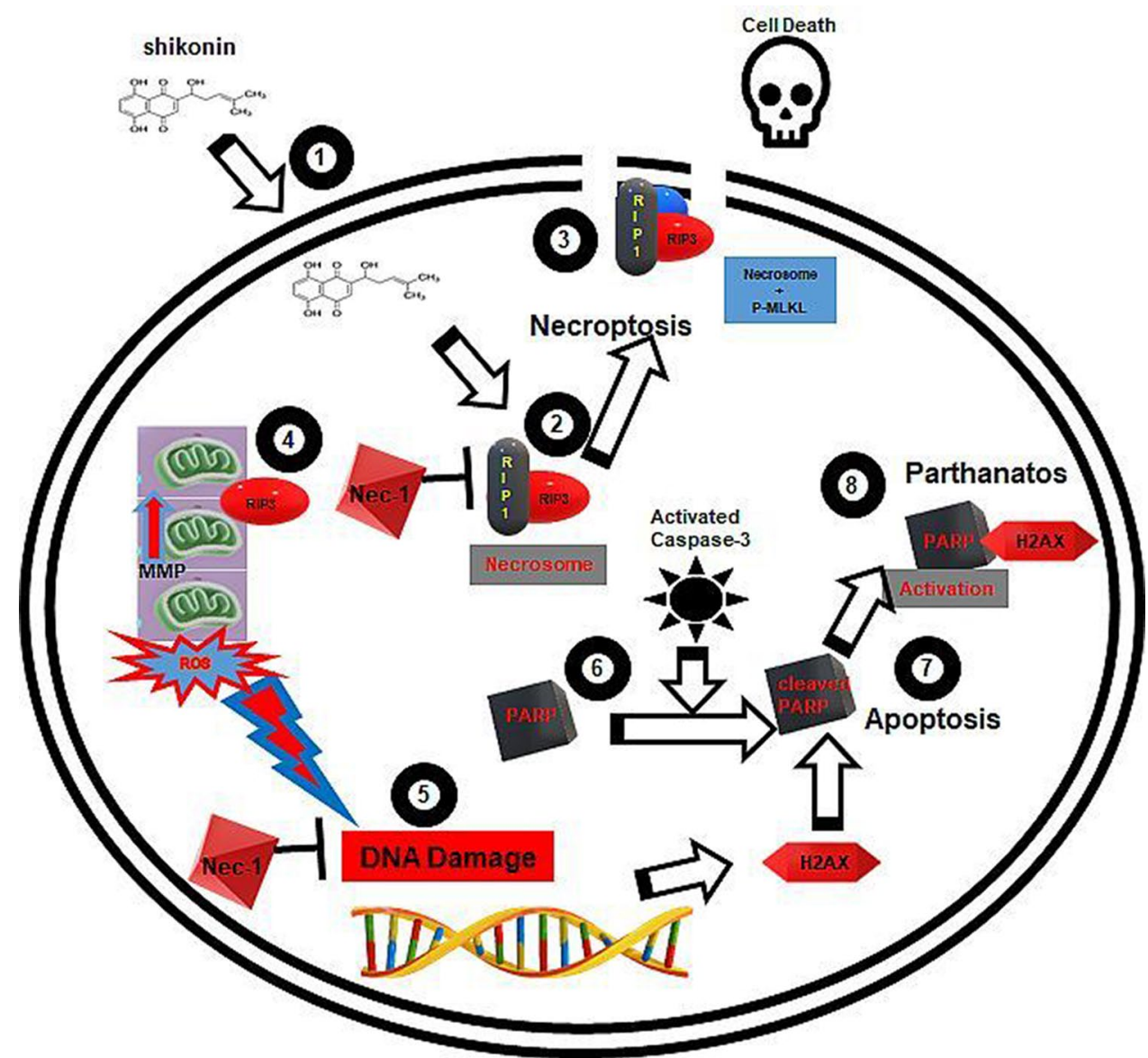


cleaved PARP which zVAD can down-regulate Fig. 1 [8, 19]. The action of cleaved PARP is also known to reduce levels of NAD + resulting in lower ATP levels which has been reported to act as a molecular switch towards programmed necrosis [15]. RIP1 is not only located in the cytoplasm which together with RIP3 forms the necrosome, but parthanatos is also found in the nucleus associated with activated PARP1 and RIP1 in the development of a parthanatos response [19-21]. RIP3 has also been reported to regulate levels of mitochondrial ROS in glioma cells which in turn increased the incidence of double strand breaks in DNA (DNA DSBs) leading to an increase in $\gamma \mathrm{H} 2 \mathrm{AX}$ or DNA Damage resulting in chromatinolysis and necroptosis [8]. Jurkat cells have previously been shown to display no change in the high level of DNA Damage in live necroptotic cells (reduced in dead cells) after treatment with shikonin [18]. Shikonin treatment of Jurkat cells were also reported to show increased hyper-activation of PARP by $\gamma \mathrm{H} 2 \mathrm{AX}$ and cleaved PARP in live populations as well as dead necroptotic and oncotic cells (RIP3 ${ }^{-v e} /$ Caspase- $3^{-v e}$ ) $[15,16,18]$. Thus live necroptotic cells are composed of different sub-populations of cells undergoing caspase-3 independent cleaved PARP, parthanatos, DNA Damage or just RIP3 up-regulated necroptosis $[15,16,18]$.

Mitochondrial activity during the necroptotic process has been misreported by the misuse of mitochondrial functional probes without using cell viability probes when performing flow cytometry $[14,22]$. The non-exclusion of dead cells induced by the cytotoxic drug in question leads to a massive under-reporting of the degree of MMP in the remaining live cells. This study investigates this discrepancy by using a cell viability probe to show changes in MMP and ROS generation in live cells undergoing necroptosis as well as oncosis, early, late and RIP1-dependent apoptosis and relates this to parthanatos, cleaved PARP expression and the degree of DNA Damage during RCD and ACD (Accidental Cell Death) processes. Further studies investigating the modulation of such responses to shikonin by blockade of necroptosis or apoptosis by Nec-1 or zVAD were also performed. This flow cytometric approach in the study of multiple forms of RCD and ACD highlights the presence of functional hyperpolarized mitochondria with high levels of ROS in live and apparently dead necroptotic cell populations.

\section{Materials and methods}

\section{Induction of necroptosis and apoptosis}

Jurkat cells (human acute T cell leukaemia cell line) were grown in RPMI 1640 medium with $10 \%$ FBS and penicillin/streptomycin (Invitrogen, UK) at $37{ }^{\circ} \mathrm{C}$ and $5 \% \mathrm{CO}_{2}$ and either left untreated or treated with $0.5 \mu \mathrm{M}$ Shikonin (Santa
Cruz, USA) for $24 \mathrm{~h}$. Cells were also pre-treated with the pan-caspase blocker zVAD $(20 \mu \mathrm{M}$, Enzo Life Sciences, USA) or the necroptosis blocker necrostatin-1 $(60 \mu \mathrm{M}$, Cambridge Bioscience, UK) for $2 \mathrm{~h}$ before and during the treatment with $0.5 \mu \mathrm{M}$ Shikonin for $24 \mathrm{~h}$.

\section{Live cell labelling}

After treatment part of the harvested cells $\left(1 \times 10^{6}\right)$ were labelled with Violet Live Cell Caspase $(2 \mu 1 / \mathrm{ml}$, Becton Dickinson, USA) and $500 \mathrm{nM}$ CellROX Green (Invitrogen, USA) at $37{ }^{\circ} \mathrm{C}$ for $1 \mathrm{~h}$. Then incubated with $50 \mathrm{nM}$ MitoTracker Deep Red (Invitrogen, USA) and fixable live dead stain Zombie NIR (Near Infra-Red) (BioLegend, UK) at $37{ }^{\circ} \mathrm{C}$ for $15 \mathrm{~min} .100,000$ events were analysed on an ACEA Bioscience Novocyte 3000 flow cytometer.

\section{Intracellular labelling protocol}

The remaining Jurkat cells were labelled with fixable live dead stain Zombie NIR for 15 min at RT and washed. Pelleted cells were sequentially fixed in Solution A (CalTag, UK) then permeabilised with $0.25 \%$ Triton X-100 (Sigma, UK) for 15 min each at RT. Jurkat cells $\left(2 \times 10^{6}\right)$ were incubated for $20 \mathrm{~min}$ at RT with $2 \mu \mathrm{l}$ of anti-RIP3-PE (clone B-2, Cat. No. sc-374639, Santa Cruz, USA), cleaved anti-PARPPE-CF594 (clone F21-852, Becton Dickinson, USA), anti$\gamma \mathrm{H} 2 \mathrm{AX}$-PE-Cy7 (clone 2F3, BioLegend, UK) and antiactive caspase-3-BV650 (clone C92-605, Becton Dickinson, USA) for $20 \mathrm{~min}$ at RT. Washed cells were resuspended in $400 \mu \mathrm{l}$ PBS and analysed on a ACEA Bioscience Novocyte 3000 flow cytometer and 200,000 events analysed.

\section{Flow cytometry}

MitoTracker Deep Red and Zombie NIR were excited by the $633 \mathrm{~nm}$ laser and collected at 660/20 nm and 780/60 nm. Caspase Violet and Caspase-3-BV650 were excited by the $405 \mathrm{~nm}$ laser and collected at 450/20 nm and 675/30 nm. CellROX Green, RIP3-PE, cleaved PARP-PE-CF594, $\gamma \mathrm{H} 2 \mathrm{AX}-\mathrm{PE}-\mathrm{Cy} 7$ were excited by the $488 \mathrm{~nm}$ laser and collected at 530/20 nm, 572/28, 615/20, 780/60 nm respectively. Single colour controls were employed to determine the colour compensation using the pre-set voltages on the instrument using Novo Express software (ver 1.2.5, ACEA Biosciences, USA).

\section{Gating strategies}

Unfixed cells were gated on FSC vs SSC removing the small debris near the origin with single cells gated on an FSC-A vs FSC-H dot-plot. This was followed by a dot-plot of Caspase-Violet vs Zombie NIR with a quadrant placed 
marking off live cells in the double negative quadrant (lower left), early apoptotic cells were Caspase Violet ${ }^{+\mathrm{ve}} /$ Zombie $\mathrm{NIR}^{-\mathrm{ve}}$ (lower right) and lastly with dead cells labelled with

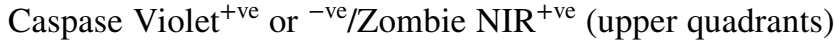
indicating late apoptotic and necrotic/oncotic cells respectively (Fig. 1S). Live, early and late apoptotic and oncotic populations were gated for CellROX Green vs MitoTracker Deep Red with upper-left quadrant CellROX Green $^{-v e}$ / MitoTracker Deep $\mathrm{Red}^{+\mathrm{ve}}$ or live cells with mitochondrial function but without ROS. The upper right quadrant being CellROX Green ${ }^{+v e} /$ MitoTracker Deep Red $^{+v e}$ or cells with mitochondrial function and ROS, lower right quadrant being CellROX Green ${ }^{+v e} /$ MitoTracker Deep Red ${ }^{-v e}$ or cells without mitochondrial function but with ROS (Fig. 2S). MitoTracker Deep Red and CellROX Green signals from shikonin treatments were referenced to untreated cells (100\%) then expressed as percentage fold increase, see Fig. 5.

After Zombie NIR labelling, fixation, permeabilisation and intracellular labelling with antibodies (listed in the "Intracellular labelling protocol" section). Cells were then analysed by gating on a dot-plot of Caspase-3-BV650 vs Zombie NIR with a quadrant placed marking off live cells in the double negative quadrant (lower left), with Caspase-3-BV650 ${ }^{+v e} / Z_{\text {Zombie NIR }}{ }^{-v e}$ (lower right) indicating early apoptotic (EAPO) cells (Fig. 3S, a). Lastly, Caspase-3-BV650 ${ }^{+v e}$ or -ve $/ Z o m b i e ~ N_{R}^{+v e}$ upper quadrants indicated late apoptotic (LAPO) and oncotic cells, respectively (Fig. 3S, a). Live (including early apoptotic) and both dead cell populations were gated separately and analysed in RIP3 vs Caspase-3 dot-plots with RIP $3^{+v e} /$ Caspase- $3^{-v e}$ which indicated live cells or necroptosis when RIP3 Median Fluorescence Intensity (MFI) was up-regulated (Fig. 3S, b, d). RIP ${ }^{-v e} /$ Caspase- $3^{+v e}$ cells indicate those that had undergone early (EAPO) or late apoptosis (LAPO, Fig. 3S, b, d). Double positive events indicate cells of the RIP1-dependent apoptosis phenotype (RIP1APO, Fig. 3S, b, d). Double negative (DN) cells and all other phenotypes were further analysed for $\gamma \mathrm{H} 2 \mathrm{AX}$ and cleaved PARP expression (Fig. 3S, c, e). The following populations were identified: DNA Damage (DDR) phenotype were $\gamma \mathrm{H} 2 \mathrm{AX}^{+\mathrm{ve}} / \mathrm{PARP}^{-\mathrm{ve}}$ events, $\gamma \mathrm{H} 2 \mathrm{AX}$ hyper-activation of cleaved PARP (Hyper-Act) or in the absence of caspase-3, parthanatos $\gamma \mathrm{H} 2 \mathrm{AX}^{+\mathrm{ve}} / \mathrm{PARP}^{+\mathrm{ve}}$, cleaved PARP (PARP) as $\gamma \mathrm{H} 2 \mathrm{AX}^{-\mathrm{ve}} / \mathrm{PARP}^{+\mathrm{ve}}$ and negative (QN, $\gamma \mathrm{H} 2 \mathrm{AX}^{-\mathrm{ve}} / \mathrm{PARP}^{-\mathrm{ve}}$ ) as shown in Fig. 3S, c, e.

\section{Statistics}

All experiments (at least $n=3$ ) were expressed either as average percent positive \pm SD, average Median Fluorescence Intensity $(\mathrm{MFI}) \pm \mathrm{SD}$ and percent average Median Fluorescence Intensity (MFI) normalised to untreated cells \pm SD. Data were tested for multivariate normality with the Kolmogorov-Smirnov goodness of fit test, followed by
One Way ANOVA $(P<0.5)$ and then post ad hoc to test for significance $(P<0.5)$ between treatments with GraphPad PRISM ver. 8.4.0 software Inc., USA. Not considered significant $P>0.05$ (NS), significance was either $P<0.05^{*}$, $P<0.01 * *, P<0.001 * * *, P<0.0001 * * * *$ when treated cells were compared to untreated and also between treatments when analysing $\gamma \mathrm{H} 2 \mathrm{AX}$ and PARP.

\section{Results}

\section{Induction of necroptosis and apoptosis}

Jurkat cells treated with shikonin showed a significant increase in early (68\%) and late apoptosis (22\%, Fig. 2a, b). Shikonin induced necroptosis (RIP $3^{\text {high+ve }} /$ Caspase- $3^{-v e}$ ) with an up-regulation of RIP3 (37\%) and RIP1-dependent apoptosis $\left(29 \%\right.$, RIP3 ${ }^{+v e} /$ Caspase- $\left.3^{+v e}\right)$ compared to untreated live cells (Figs. 3a, c and 4). The dead cells showed increased levels of RIP1-dependent apoptosis compared to untreated cells (58\%, Fig. 3b, d).

Treated live cells were comprised of those undergoing necroptosis (4\%, RIP3 ${ }^{\text {high+ve }} /$ Caspase- $3^{-v e}$ ) or were double negative (DN) for RIP3/Caspase-3 (14\%) together which showed a significant two and four fold increase in MMP and ROS respectively compared to untreated live cells (Figs. 2S, $\mathrm{a}, \mathrm{e} ; 3 \mathrm{c}$ and $5 \mathrm{a}, \mathrm{b}$ ). There was also a rise in live cells that lacked mitochondrial function but showed no significant rise in ROS (13\% compared to untreated 2\%, Figs. 2S, a, e and 5c). Early apoptotic treated cells mainly lacked MMP (while untreated did not) but generated $>60 \%$ more ROS (Figs. 2S, b, f and 5c). The remaining treated early apoptotic cells with mitochondrial function (7\%) generated more ROS $(50 \%)$ than untreated early apoptotic cells (Figs. 2S, b, f and $5 \mathrm{a}, \mathrm{b})$.

Late apoptotic cells mainly lacked mitochondrial function, but generated $23 \%$ more ROS than untreated cells (Figs. 2S, c, g and 5c). However, the small percentage of late apoptotic cells (2\%) with raised MMP (40\%) with a 6.5fold increase in ROS (Figs. 2S, c, g and 5a, b). Oncotic cells $\left(\right.$ Zombie $^{+v e} /$ Caspase violet ${ }^{-v e}$ ) which comprised of dead necroptotic and dead oncotic DN cells showed a high degree of mitochondrial function (30\% compared to untreated cells $5 \%$ ) with raised MMP (3.6 fold) and eightfold-ninefold more ROS than untreated oncotic cells (Figs. 2S, d, h and $5 \mathrm{a}, \mathrm{b})$. Those oncotic cells without mitochondrial function showed no increase in detectable ROS (Figs. 2S, d, 5c).

Live cells showed a small increase in the incidence of $\gamma \mathrm{H} 2 \mathrm{AX}$ hyper-activation of cleaved PARP or parthanatos in live necroptotic, RIP1-dependent apoptosis and also late apoptotic cells, with a decrease observed in early apoptotic cells (Fig. 6a). The incidence of cleaved PARP was significantly increased after shikonin treatment in early, live 
Fig. 2 Cell death and caspase-3 activation assay. Jurkat cells were untreated (a), treated with $0.5 \mu \mathrm{M}$ shikonin for $24 \mathrm{~h}$ (b), pre-treated with $20 \mu \mathrm{M}$ $\mathrm{zVAD}$ for $2 \mathrm{~h}$ then with $0.5 \mu \mathrm{M}$

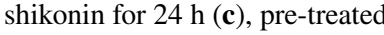
with $60 \mu \mathrm{M}$ necrostatin-1 (Nec1) for $2 \mathrm{~h}$ then with $0.5 \mu \mathrm{M}$ shikonin for $24 \mathrm{~h}(\mathbf{d})$. Live (DN), early apoptotic (EAPO, Zombie NIR ${ }^{-v e} /$ caspase $3^{+v e}$, late apoptotic (LAPO, Zombie $\mathrm{NIR}^{+\mathrm{ve}} /$ caspase $3^{+\mathrm{ve}}$ ) and oncotic cells (Zombie NIR ${ }^{+\mathrm{ve}}$ / caspase $\left.3^{-v e}\right)$. Average $\% \pm \mathrm{SD}$ $(n=3)$ were analysed for significance by One Way ANOVA $(P<0.5)$ and post ad hoc tested for significance, NS $(P>0.5$, not significant), significance was $P<0.05^{*}, P<0.01 * *$, $P<0.001 * * *, P<0.0001 * * * *$, with red arrows indicating change compared to untreated cells (Color figure online) a Untreated cells

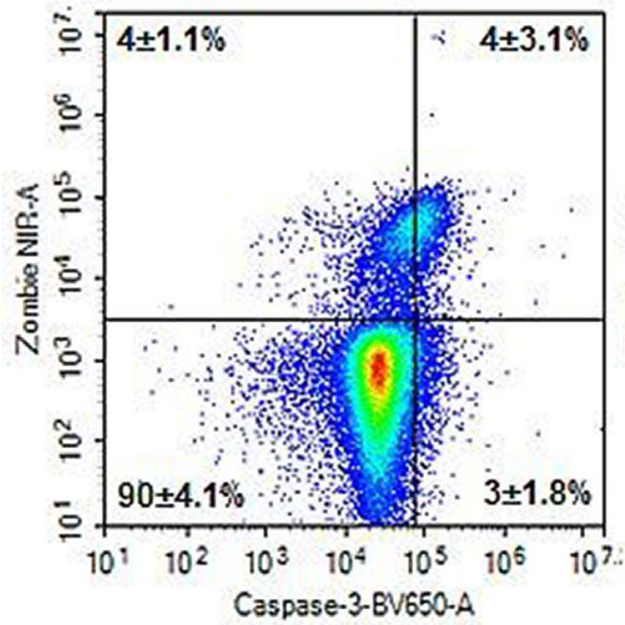

c +zVAD/0.5 $\mu \mathrm{M}$ shikonin

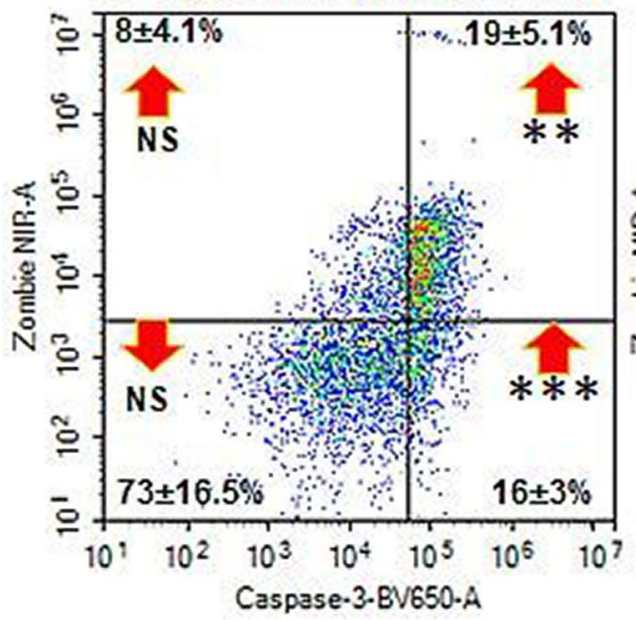

b $0.5 \mu \mathrm{M}$ shikonin

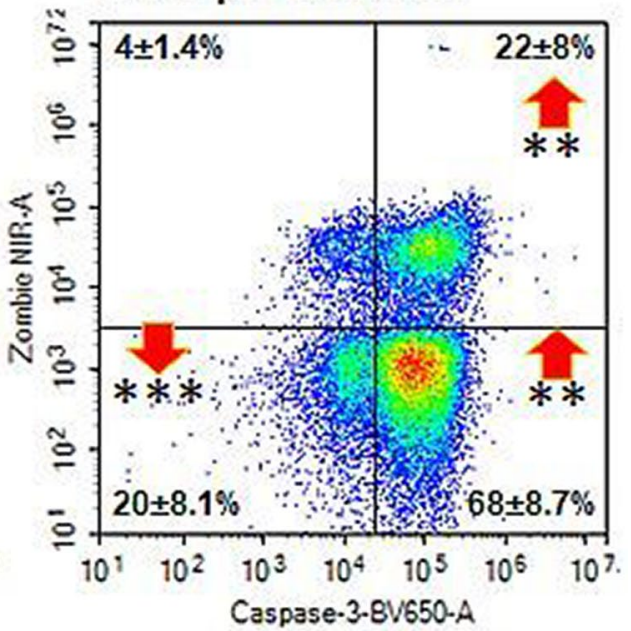

d Nec-1/0.5 $\mu \mathrm{M}$ Shikonin

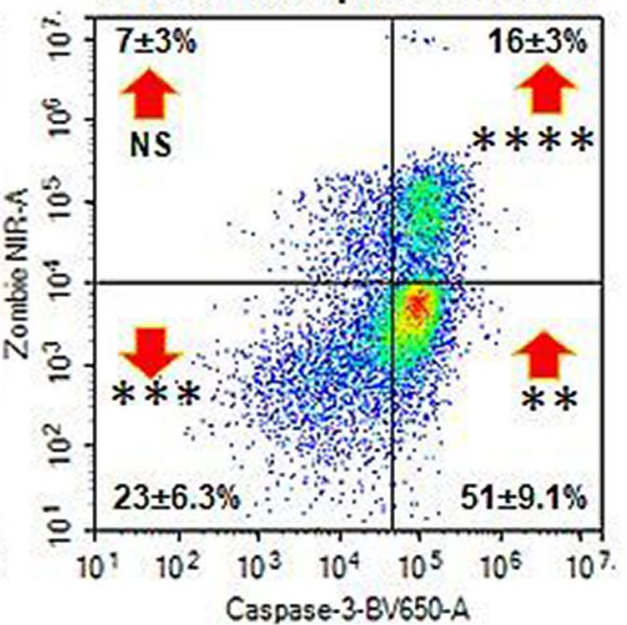

RIP1-dependent apoptosis and both necroptotic populations; with decreases in late apoptosis and dead RIP1-dependent apoptosis (Fig. 6b). DNA Damage was correspondingly decreased after drug treatment in both necroptotic populations and live RIP1-dependent apoptosis, with an increase observed in late apoptotic cells (Fig. 6c).

\section{zVAD blockade of apoptosis}

Blockade of shikonin induced apoptosis by zVAD showed a decrease in early apoptosis (16\%, Fig. 2c). The incidence of live necroptotic cells with raised RIP3 MFI was increased (14\% compared to $4 \%$ treated cells, Figs. 3e and 4). Early/ late and RIP1-dependent apoptosis was reduced compared to drug treatment (Figs. 3e, f and 4). Correspondingly there was a significant increase in live and dead oncotic DN populations compared to drug and untreated cells (Fig. 3a-f).
The live cells after zVAD blockade of shikonin comprised of $14 \%$ necroptotic and $53 \%$ DN compared to $4 \%$ and $14 \%$ in treated cells respectively (Fig. 3c, e). These live cells showed a doubling of cells without mitochondrial function which generated ROS (Figs. 2S, i and 5c). Consequently, the incidence of double positive live cells was reduced $(56 \%)$ compared to treated cells $(70 \%)$ but had the same high level of MMP as that induced by shikonin treatment (two fold increase) and ROS (fourfold increase, Figs. 2S, i and 5a, b). Early and late apoptotic cells after zVAD blockade of shikonin showed an abrogation of ROS generated by cells with functioning mitochondria with no change in the level of ROS in cells without mitochondrial function compared to drug treatment (Figs. 2S, b, c, f, g, j, k and 5). The incidence of apparently live oncotic cells after zVAD blockade was reduced to untreated levels but with the maintenance of the high level of MMP and ROS (Figs. 2S, d, h, 1 and 5a, b). However, the incidence of cells without mitochondrial 

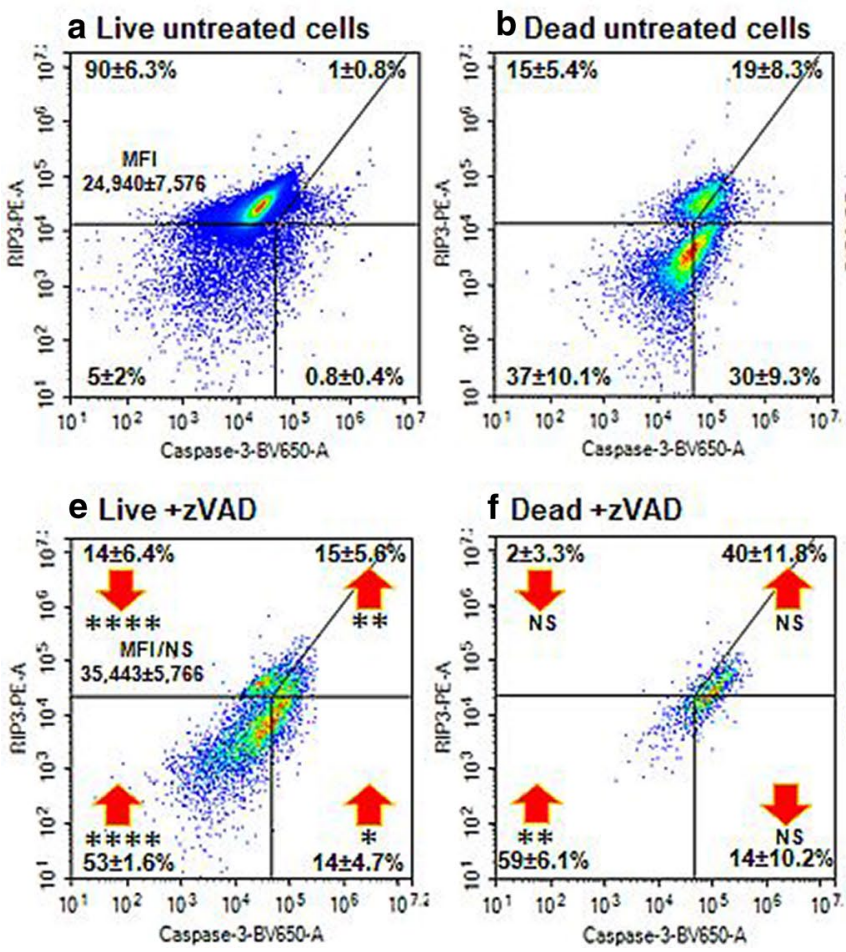

Fig. 3 RIP3 and caspase-3 activation assay. After gating on live and dead cells from a Zombie NIR vs. Caspase-3-BV650 dot-plot cells were then analysed on a RIP3-PE vs. Caspase-3-BV650 dot-plot. The necroptotic phenotype indicated by RIP $3^{+v e} /$ high $^{\text {ve }} /$ Caspase- $3^{-v e}$ which has up-regulated RIP3 Median Fluorescent Intensity (MFI). The apoptotic phenotype was RIP ${ }^{-v e} /$ Caspase- $3^{\text {+ve }}$, RIP1-dependent apoptosis (RIP3 ${ }^{+\mathrm{ve}} /$ Caspase- $3^{+\mathrm{ve}}$ ) and quadruple negative $\left(\mathrm{RIP} 3^{-\mathrm{ve}} /\right.$ Caspase- $3^{-v e}$. Live (a) and dead (b) untreated cells, or cells treated with $0.5 \mu \mathrm{M}$ shikonin for $24 \mathrm{~h}(\mathbf{c}, \mathbf{d})$, pre-treated with $20 \mu \mathrm{M} \mathrm{zVAD}$

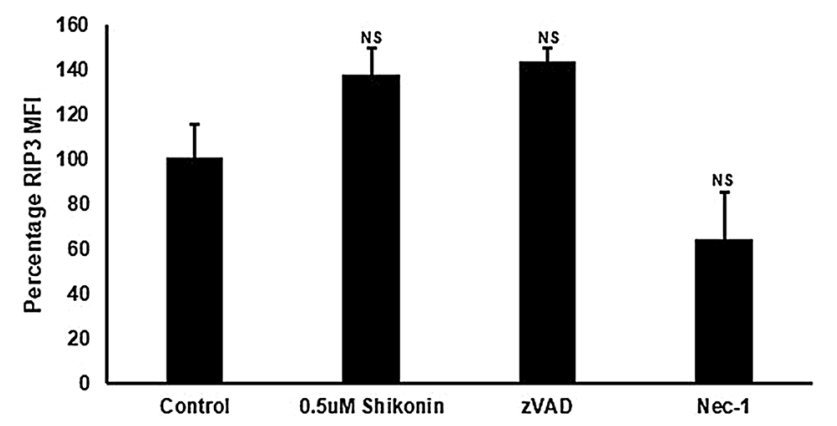

Fig.4 Analysis of RIP3 expression. Live cells expressing RIP3 but not caspase-3 were analysed for RIP3 expression for untreated cells, then average percentage in RIP3 MFI \pm SD were determined for $0.5 \mu \mathrm{M}$ shikonin $(24 \mathrm{~h}), 0.5 \mu \mathrm{M}$ shikonin with $20 \mu \mathrm{M}$ zVAD or $0.5 \mu \mathrm{M}$ shikonin with $60 \mu \mathrm{M} \mathrm{Nec-1.} \mathrm{Average} \mathrm{percentage}$ of RIP3 MFI $\pm \mathrm{SD}(\mathrm{n}=3)$, were analysed for significance by One Way ANOVA $(P<0.5)$ and post ad hoc tested for significance, NS $\left(P=>0.5\right.$, not significant), significance was $P<0.05^{*}, P<0.01^{* *}$, $P<0.001^{* * *}, P<0.0001 * * * *$ compared to untreated cells
C Live shikonin

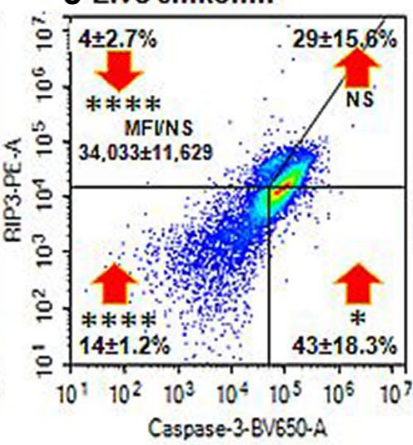

g Live + Nec- 1

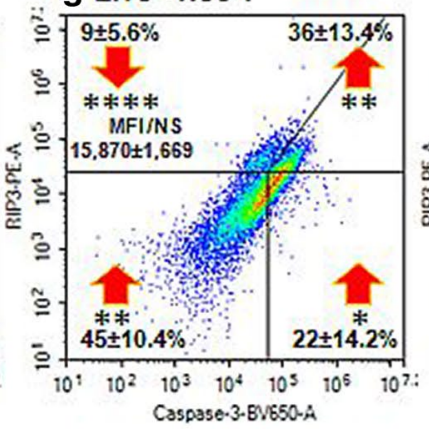

d Dead shikonin

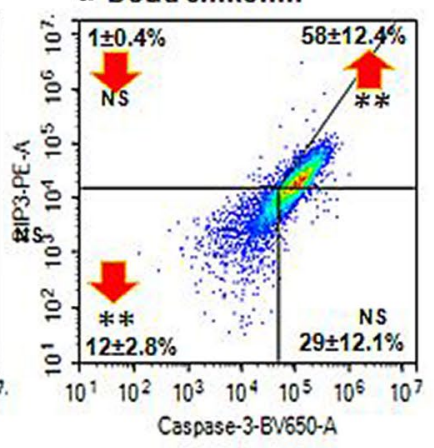

h Dead +Nec-1

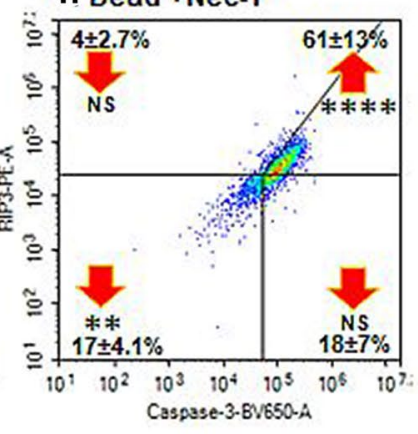

for $2 \mathrm{~h}$ then with $0.5 \mu \mathrm{M}$ shikonin for $24 \mathrm{~h}(\mathbf{e}, \mathbf{f})$ or pre-treated with $60 \mu \mathrm{M} \mathrm{Nec}-1$ for $2 \mathrm{~h}$ then with $0.5 \mu \mathrm{M}$ shikonin for $24 \mathrm{~h}(\mathbf{g}, \mathbf{h})$. Average Median Fluorescent Intensity MFI or average $\% \pm$ SD $(n=3)$ were analysed for significance by One Way ANOVA $(P<0.5)$ and post ad hoc tested for significance, NS $(P>0.5$, not significant $)$, significance was $P<0.05^{*}, P<0.01^{* *}, P<0.001^{* * *}, P<0.0001^{* * * *}$, with red arrows indicating change compared to untreated cells (Color figure online)

function but generated the same level of ROS was increased (15\%) compared to drug and untreated cells ( $<5 \%$, Figs. $2 \mathrm{~S}$, d, h, 1 and 5c).

$\gamma \mathrm{H} 2 \mathrm{AX}$ hyper-activation of cleaved PARP or parthanatos and cleaved PARP was reduced in most cell populations after zVAD blockade of shikonin compared to drug treatment except live necroptotic cells which showed an increase in cleaved PARP (Fig. 6a, b). In contrast the DNA Damage populations was increased in most cell populations after zVAD blockade of shikonin compared to drug treatment (Fig. 6c).

\section{Necrostatin-1 blockade of necroptosis}

Nec-1 blockade of shikonin reduced early apoptosis $(51 \%)$ compared to drug treatment $(68 \%$, Fig. 2 d). Such treatment blocked necroptosis as indicated by the abrogation of RIP3 up-regulation (Figs. $3 g$ and 4). While, late apoptosis was reduced, the incidence of the live DN population ( $45 \%$ ) was increased compared to drug treatment (14\%, Fig. 3c, d, g, h). 

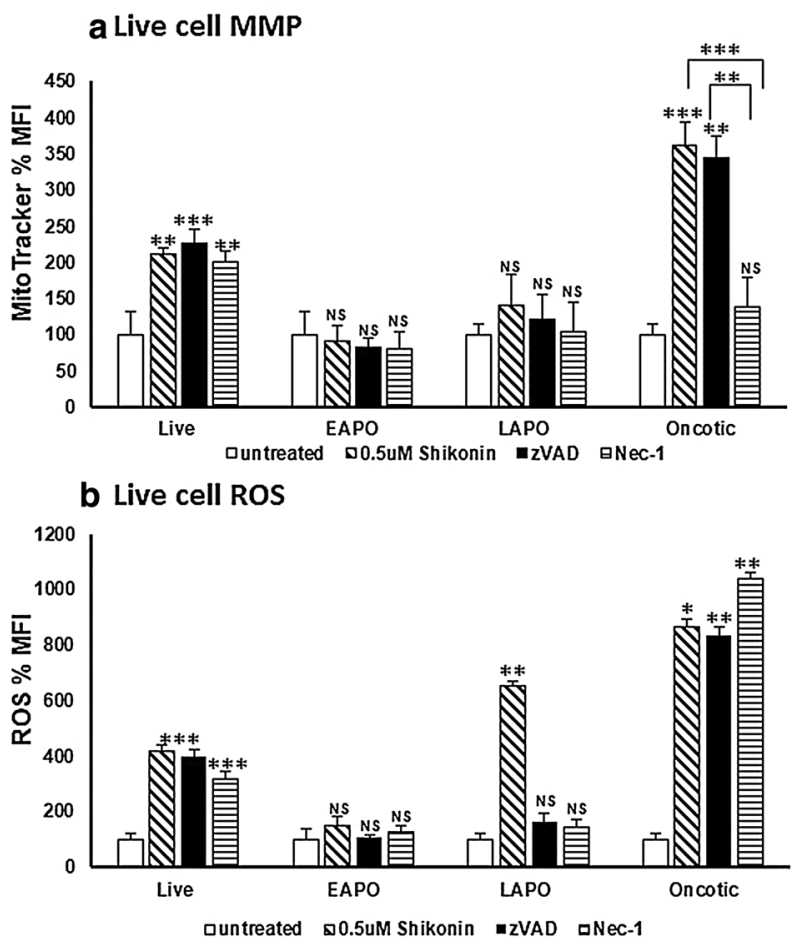

C ROS in MMP negative cells

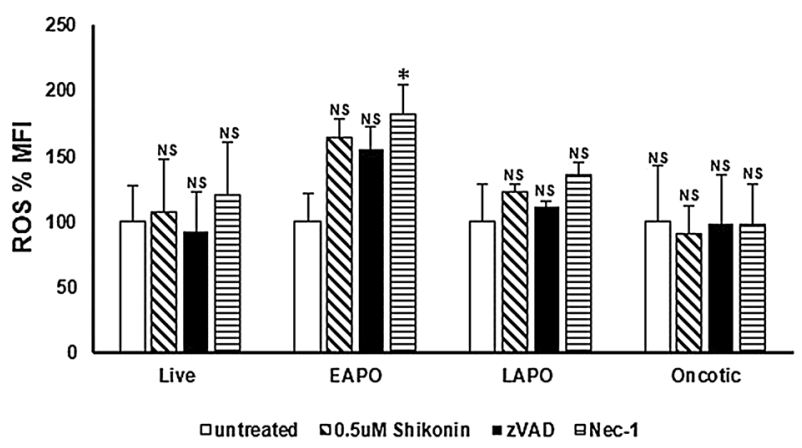

Fig. 5 Mitochondrial MMP, function and cellular ROS analysis. Jurkat cells were loaded with violet caspase, Zombie NIR, MitoTracker Deep Red and CellROX Green as described in the Materials and Methods section. Live (DN), early apoptotic (EAPO, Zombie NIR ${ }^{+\mathrm{ve}}$ / caspase violet ${ }^{+\mathrm{ve}}$ ), late apoptotic (LAPO, Zombie $\mathrm{NIR}^{+\mathrm{ve}} /$ caspase violet $^{+\mathrm{ve}}$ ) and oncotic (Zombie NIR ${ }^{+\mathrm{ve}} /$ caspase violet $^{-\mathrm{ve}}$ ) cells were analysed for MMP by MitoTracker Deep Red (a), those MMP + ve cells analysed for CellROX Green, ROS (b), those MMP-ve cells analysed for CellROX Green, ROS (c). Untreated cells, $0.5 \mu \mathrm{M}$ shikonin, blockade of shikonin with zVAD $(20 \mu \mathrm{M})$ or blockade of shikonin with Nec-1 $(60 \mu \mathrm{M})$ were analysed. Mean percentage of MFI was normalised to untreated cells $\pm \mathrm{SD},(\mathrm{n}=3)$ were analysed for significance by One Way ANOVA $(P<0.5)$ and post ad hoc tested for significance, NS $\left(P>0.5\right.$, not significant), significance was $P<0.05^{*}$, $P<0.01^{* *}, P<0.001^{* * *}, P<0.0001^{* * * *}$ compared to untreated cells and between treatments as indicated
This increased the percentage of live DN cells $(45 \%$, Fig. $3 \mathrm{~g}$ ) resulted in a reduction in live cells with functioning mitochondria (49\% compared to $70 \%$ by drug treatment) but which still displayed increased MMP and ROS by 2 and threefold respectively (Figs. 2S, a, e, m and 5a, b). Early apoptotic cells showed no change in the degree of ROS generation in cells with or without mitochondrial function (Figs. 2S, b, c, n, o and 5). While, late apoptotic cells with dysfunctional mitochondria showed no significant rise in generated ROS levels above untreated cells, the few cells with functional mitochondria showed an abrogation of ROS (Fig. 4b, c). Oncotic cells with dysfunctional mitochondria showed no change in ROS after Nec-1 blockade, while those with functioning mitochondria were reduced $(<4 \%)$ and showed an abrogation of the raised MMP $(>40 \%)$ but had a ten fold increase in ROS compared to untreated cells (Figs. 2S, d, p and 5a, b, c).

The incidence of $\gamma \mathrm{H} 2 \mathrm{AX}$ hyper-activated cleaved PARP expressing cells was reduced after Nec-1 blockade of shikonin in only early and live RIP1-dependent apoptotic populations compared with drug treatment, with no change observed in other populations (Fig. 6a). Cleaved PARP was increased by Nec-1 in late apoptotic, live necroptotic and dead oncotic DN cells compared to drug treatment, with a reduction observed in the early apoptotic population (Fig. 6b). In contrast, all cells after Nec-1 blockade showed reductions in DNA Damage as opposed to the increase observed after zVAD blockade of shikonin (Fig. 6c).

\section{Discussion}

The main aim of this flow cytometric based study was to show that shikonin induced Jurkat cell necroptosis and apoptosis (blocked by zVAD and Nec-1) resulted in measurable changes in mitochondrial function, MMP and cellular ROS in live, early, late apoptotic and oncotic cell populations. Unfortunately, the lack of reliable MitoTracker and CELLROX Green data after fixation and permeabilisation has not allowed analysis of MMP and ROS in specific cell death populations (e.g. live necroptotic cells undergoing parthanatos). The assay also tracked the incidence of shikonin (blocked by zVAD or Nec-1) induced necroptosis, caspase-3 dependent apoptosis, RIP1-dependent apoptosis, DN populations (live and dead oncotic DN cells) as well as the incidence of parthanatos (or $\gamma \mathrm{H} 2 \mathrm{AX}$ hyper-activation of PARP), cleaved PARP and DNA Damage in these populations. Other studies imply that the necroptosis process is typified by the presence of dysfunctional mitochondria and high levels of ROS, this was mainly due to the misreporting of MitoTracker data due to the lack of a cell viability probe [14, 22]. Cytotoxic drugs usually cause a high degree of cell death with 
Fig. $6 \gamma \mathrm{H} 2 \mathrm{AX}$ hyper-activation of cleaved PARP or parthanatos, cleaved PARP and $\gamma \mathrm{H} 2 \mathrm{AX}$ or DNA Damage assay. Jurkat untreated, treated with $0.5 \mu \mathrm{M}$ shikonin or pre-treated $\mathrm{zVAD}$ $(20 \mu \mathrm{M})$ or Necrostatin-1 $(60 \mu \mathrm{M})$ for $2 \mathrm{~h}$ then incubated with $0.5 \mu \mathrm{M}$ shikonin for $24 \mathrm{~h}$. After gating on live and dead cells from a Zombie NIR vs. Caspase-3-BV650 dot-plot untreated or treated live and dead Jurkat cells were analysed on a RIP3-PE v Caspase3-BV650 dot-plot. From which early and late apoptotic, necroptotic, RIP1-dependent apoptotic and double negative (DN) populations were analysed for $\gamma \mathrm{H} 2 \mathrm{AX}$ and cleaved PARP, see Figs. 1S and $2 S$ for detailed information. The incidence of $\gamma \mathrm{H} 2 \mathrm{AX}$ hyper-activation of cleaved PARP or parthanatos (a), cleaved PARP (b) and DNA Damage (c) were determined for all populations listed above. Average $\% \pm \mathrm{SD},(\mathrm{n}=3)$ were analysed for significance by One Way ANOVA $(P<0.5)$ and post ad hoc tested for significance, NS ( $P>0.5$, not significant), significance was $P<0.05^{*}$, $P<0.01^{* *}, P<0.001^{* * *}$, $P<0.0001 * * * *$ compared to untreated cells and between treatments as indicated
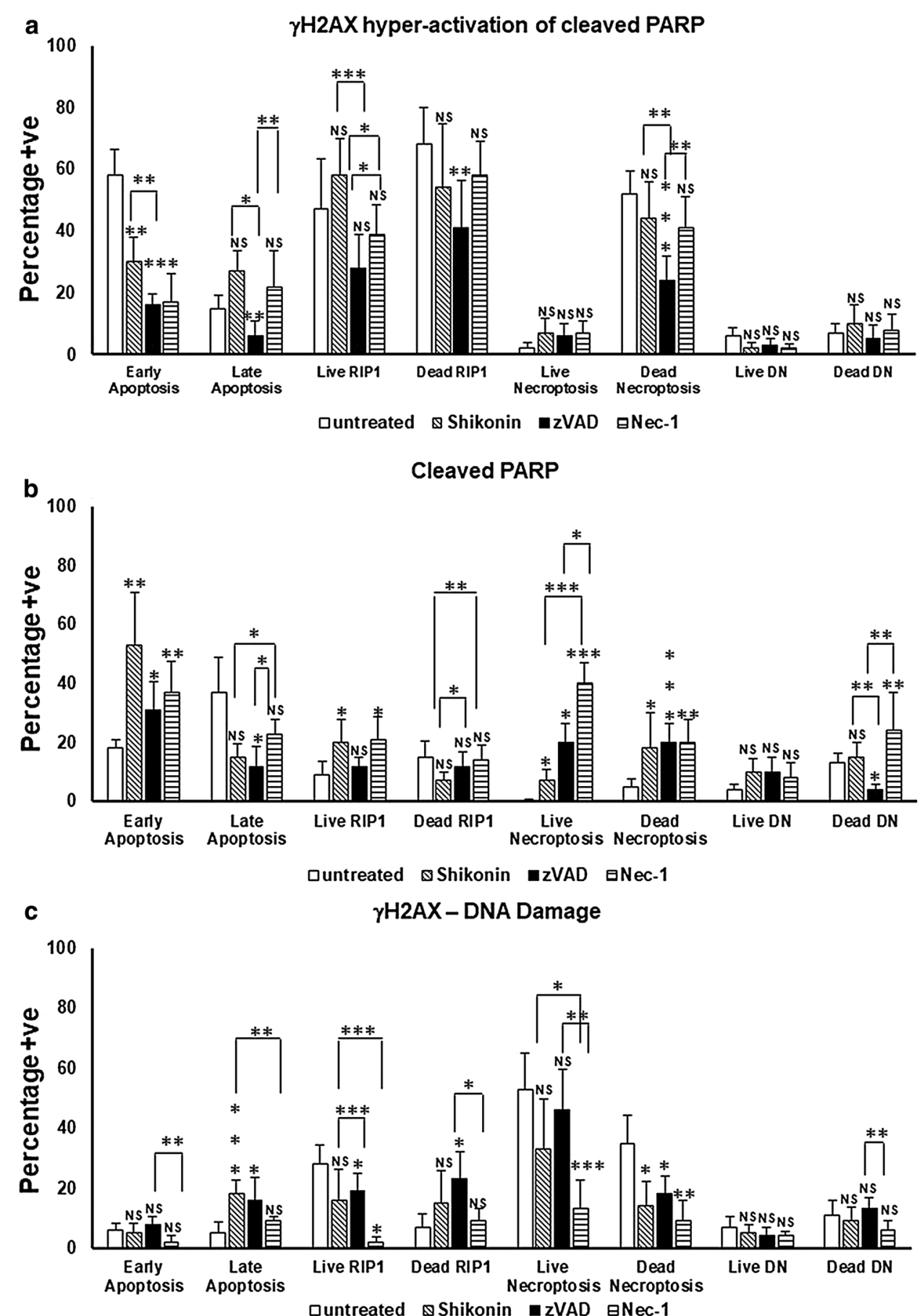

the possibility that the remaining live cells (with functioning mitochondria) are thus hidden by the dead cell population (without functioning mitochondria) leading to a misreporting of the health of mitochondria within the live cell fraction [14]. Necroptosis occurs over a period of time and the high level of ROS being detected is due at some point to the mitochondria in live necroptotic cells being functional and in a hyper-polarized state leading to the generation of most of the ROS detected Fig. 1 [2, 14, 21-24]. Other intracellular sources of ROS have been shown to be less affected by blockade with Nec-1 (unlike mitochondrial generated ROS) indicating that a small but significant proportion of ROS is not generated by mitochondria [8]. Although this does not indicate an absolute mitochondria requirement in the necroptotic process [2, 14, 21-24].

The use of multi-parameter flow cytometry to analyse RCD and ACD processes showed that live necroptotic cells (indicated by a 37\% up-regulation of RIP3 which was abrogated by Nec-1) had functioning mitochondria with high levels of MMP and ROS which can be divided into 
the basic necroptotic phenotype which were negative for both $\gamma \mathrm{H} 2 \mathrm{AX}$ and cleaved PARP, while a high proportion of the necroptotic population displayed DNA Damage which was not increased by the high levels of ROS in these cells as may have been expected, see pathway of ROS induction of DNA Damage Fig. 1 [8, 14, 19]. The shikonin induced necroptosis within the live cell fraction also generated at a low incidence two more definable necroptotic populations which displayed cleaved PARP and parthanatos respectively, see pathway in Fig. 1 [8, 14, 19]. Early, late and RIP1-dependent apoptotic cells had little mitochondrial function but such early and live RIP1-dependent apoptotic cells showed increased ROS compared to untreated cells which was abrogated by zVAD. Early apoptotic and live RIP1-dependent apoptotic cells showed increased cleaved PARP (reduced by zVAD), with DNA Damage being reduced by Nec-1 blockade of shikonin (Fig. 1). zVAD as expected reduced levels of cleaved PARP and $\gamma \mathrm{H} 2 \mathrm{AX}$ hyper-activation of PARP in the dead apoptotic populations but increased the level of DNA Damage in dead RIP1-dependent apoptosis which Nec-1 reduced.

Once mitochondria became dysfunctional the ROS generated must have fallen relatively rapidly given that ROS levels in the live cell population without mitochondrial function have similar lower levels of ROS as those cells undergoing RIP1-dependent, early and late apoptosis. It would have been interesting to show how the level of ROS generated specifically from increasingly hyper-polarized mitochondria changed over time during the process of necroptosis and ultimately cell death. This time-course approach could also be used to monitor the effect Nec-1 blockade has on shikonin induced ROS and changes in MMP as it has been shown to significantly reduce ROS generated by mitochondria but not cellular ROS [8]. When these live necroptotic cells lose plasma membrane integrity or undergo cell death, the mitochondria in a proportion of these cells along with the live DN phenotype become more hyper-polarized (by 70\%), presumably these cells make more ATP by such activity while at the same time double the level of ROS generated. Such implied increased energy levels indicate that these 'dead' necroptotic cells could perhaps have the capacity to influence ongoing biological processes even when the cell has lost plasma membrane integrity or has undergone the classic definition of cell death. It would also be interesting to observe how these biologically hyper-active cells initially develop, mature and degrade by studying these cells over time which may give an insight into their potential to alter the outcome of RCD and ACD processes.

More of these dead necroptotic cells have also then switched from mainly a DNA Damage phenotype to a parthanatos phenotype compared to live necroptotic cells. This indicated that the high level of ROS generated by these cells resulted in the $\gamma \mathrm{H} 2 \mathrm{AX}$ hyper-activation of cleaved PARP to generate this parthanatos population of dead necroptotic cells, see pathway of ROS driving development of parthanatos in Fig. 1. While, dead oncotic DN cells did not undergo such a change in RCD phenotypes significantly even though these cells also generated high levels of ROS, see Fig. 1.

Inhibitor blockade of shikonin did not change the degree of MMP and ROS generation in the live necroptotic cell phenotype but did result in increased cleaved PARP with reductions in DNA Damage by drug and Nec-1 but not zVAD. This indicated an enhancement of DNA repair mechanisms after Nec-1 blockade of shikonin which resulted in no change in the low incidence of necroptotic cells which displayed parthanatos, see pathway in Fig. 1. Upon cell death, dead necroptotic cells after inhibitor treatment showed no change in levels of cleaved PARP compared to drug induced levels, but there were reductions in DNA Damage by Nec-1 blockade of shikonin this apparently leading to increased levels of dead necroptotic cells which displayed parthanatos (compared to live necroptotic cells), this being decreased by zVAD but unchanged by Nec-1. In contrast dead oncotic DN cells showed no significant change in the incidence of parthanatos and DNA Damage after inhibitor treatments, although Nec-1 did increase the incidence of cleaved PARP, zVAD correspondingly decreased cleaved PARP. This indicated that shikonin acted upon a proportion of live cells which resulted in a down-regulation of RIP3 (rather than up-regulation to induce necroptosis) making them the live DN phenotype, they also showed low levels of cleaved PARP and $\gamma \mathrm{H} 2 \mathrm{AX}$ but high levels of MMP and ROS. This phenotypic profile did not change significantly upon cell death even though a high proportion of these cells showed hyper-polarized mitochondria with high levels of ROS but did not result in any increase in cell death markers. Although inhibition of shikonin reduced the incidence of this population, Nec-1 reduced MMP and increased the cleaved PARP dead oncotic DN population. Why shikonin induced this differential effect upon a cell line such as Jurkat $T$ cells is difficult to ascertain but perhaps indicates that cells in a culture respond differently to the drug due to perhaps nutrient levels, functionality of organelles such as mitochondria, intracellular ROS levels and also the stage of cell division. This leads to shikonin apparently inducing more apoptosis and less detectable necroptosis with a high proportion of live cells moving to a DN phenotype which showed little sign of an RCD process analysed for in this study.

This flow cytometric based study of some of the biological functions of cells undergoing necroptosis along with the dynamic changes in the incidence of parthanatos and DNA Damage in response to shikonin and blockade of both necroptosis or apoptosis highlights the interconnectivity of numerous RCD processes. Given that necroptotic cells can also undergo parthanatos, DNA Damage or neither, perhaps these processes should no longer be classed as completely separate forms of cell death, as cells appear to move from 
displaying several specific forms of RCD to others during their response to undergoing cell death. The definition of cell death may also be required to be reviewed given that lack of plasma membrane integrity does not necessarily mean that the cell at least initially after loss of plasma membrane integrity is not biologically functional given the presence of hyper-polarized functional mitochondria.

Funding This work was supported by internal funding from Queen Mary University London.

Data Availability All data is available on request.

\section{Compliance with ethical standards}

Conflicts of interest The author states he has no conflicting or competing interests.

Open Access This article is licensed under a Creative Commons Attribution 4.0 International License, which permits use, sharing, adaptation, distribution and reproduction in any medium or format, as long as you give appropriate credit to the original author(s) and the source, provide a link to the Creative Commons licence, and indicate if changes were made. The images or other third party material in this article are included in the article's Creative Commons licence, unless indicated otherwise in a credit line to the material. If material is not included in the article's Creative Commons licence and your intended use is not permitted by statutory regulation or exceeds the permitted use, you will need to obtain permission directly from the copyright holder. To view a copy of this licence, visit http://creativecommons.org/licenses/by/4.0/.

\section{References}

1. Zhou W, Yuan J (2014) Necroptosis in health and diseases. Semin Cell Dev Biol 35:14-23

2. Marshall KD, Baines CP (2014) Necroptosis: is there a role for mitochondria? Front Physiol 5:323

3. Lu B, Gong X, Wang ZQ, Ding Y, Wang C, Luo TF, Piao MH, Meng FK, Chi GF, Luo YN et al (2017) Shikonin induces glioma cell necroptosis in vitro by ROS overproduction and promoting RIP1/ RIP3 necrosome formation. Acta Pharmacol Sin 38:1543-1553

4. Zhang Y, Su SS, Zhao S, Yang Z, Zhong CQ, Chen X, Cai Q, Yang ZH, Huang D, Wu R et al (2017) RIP1 autophosphorylation is promoted by mitochondrial ROS and is essential for RIP3 recruitment into necrosome. Nat Commun 8:14329

5. Tummers B, Green DR (2017) Caspase-8: regulating life and death. Immunol Rev 277:76-89

6. Grootjans S, Vanden Berghe T, Vandenabeele P (2017) Initiation and execution mechanisms of necroptosis: an overview. Cell Death Differ 24:1184-1195

7. Lin Y, Devin A, Rodriguez Y, Liu ZG (1999) Cleavage of the death domain kinase RIP by Caspase- 8 prompts TNF-induced apoptosis. Genes Dev 13:2514-2526

8. Zhou Z, Lu B, Wang C, Wang Z, Luo T, Piao M, Meng F, Chi G, Luo Y, Ge P (2017) RIP1 and RIP3 contribute to shikonin-induced DNA double-strand breaks in glioma cells via increase of intracellular reactive oxygen species. Cancer Lett 390:77-90

9. Galluzzi L, Vitale I, Abrams JM, Alnemri ES, Baehrecke EH, Blagosklonny MV, Dawson TM, Dawson VL, El-Deiry WS, Fulda
S et al (2012) Molecular definitions of cell death subroutines: recommendations of the Nomenclature Committee on Cell Death 2012. Cell Death Differen 19:107-120

10. Galluzzi L, Keppc O, Krautwaldf S, Kroemerb G, Linkermannf A (2014) Molecular mechanisms of regulated necrosis. Semin Cell Dev Biol 35(24):32

11. Galluzzi L, Vitale I, Aaronson SA, Abrams JM, Adam D, Agostinis P, Alnemri ES, Altucci L, Amelio I, Andrews DW et al (2018) Molecular mechanisms of cell death: recommendations of the Nomenclature Committee on Cell Death 2018. Cell Death Differ 25:486-541

12. Wang H, Sun L, Su L, Rizo J, Liu L, Wang LF, Wang FS, Wang X (2014) Mixed lineage kinase domain-like protein MLKL causes necrotic membrane disruption upon phosphorylation by RIP3. Mol Cell 54:133-146

13. Wang Z, Guo LM, Wang SC, Chen D, Yan J, Liu FX, Huang JF, Xiong K (2018) Progress in studies of necroptosis and its relationship to disease processes. Pathol Res Pract 214:1749-1757

14. Sun W, Wu X, Gao H, Yu J, Zhao W, Lu JJ, Wang J, Du G, Chen X (2017) Cytosolic calcium mediates RIP1/RIP3 complexdependent necroptosis through JNK activation and mitochondrial ROS production in human colon cancer cells. Free Radic Biol Med 108:433-444

15. Jang KH, Jang T, Son E, Choi S, Kim E (2018) Kinase-independent role of nuclear RIPK1 in regulating parthanatos through physical interaction with PARP1 upon oxidative stress. Biochim Biophys Acta Mol Cell Res 1865:132-141

16. Cho Y, McQuade T, Zhang H, Zhang J, Chan FK (2011) RIP1dependent and independent effects of necrostatin-1 in necrosis and T cell activation. PLoS ONE 6:e23209

17. Lee HL, Pike R, Chong MHA, Vossenkamper A, Warnes G (2018) Simultaneous flow cytometric immunophenotyping of necroptosis, apoptosis and RIP1-dependent apoptosis. Methods 134-135:56-66

18. Bergamaschi D, Vossenkamper A, Lee WYJ, Wang P, Bochukova E, Warnes G (2019) Simultaneous polychromatic flow cytometric detection of multiple forms of regulated cell death. Apoptosis 24:453-464

19. Artus C, Boujrad H, Bouharrour A, Brunelle MN, Hoos S, Yuste VJ, Lenormand P, Rousselle JC, Namane A, England P et al (2010) AIF promotes chromatinolysis and caspase independent programmed necrosis by interacting with histone H2AX. EMBO J 29:1585-1599

20. Nikoletopoulou V, Markaki M, Palikaras K, Tavernarakis N (2013) Crosstalk between apoptosis, necrosis and autophagy. Biochim Biophys Acta 1833:3448-3459

21. Galluzzi L, Kepp O, Kroemer G (2016) Mitochondrial regulation of cell death: a phylogenetically conserved control. Microb Cell 3:101-108

22. Chen S, Lu X, Hu B, Zhao L, Li S, Li Z, Qing X, Liu H, Xu J, Shao Z (2018) Critical contribution of RIPK1 mediated mitochondrial dysfunction and oxidative stress to compression-induced rat nucleus pulposus cells necroptosis and apoptosis. Apoptosis 23:299-313

23. Warnes G (2016) The multiplexing of assays for the measurement of early stages of apoptosis by polychromatic flow cytometry. In: Schmid I (ed) Flow cytometry select topics. On-line: InTech Open, London, pp 85-99

24. Zorova L, Popkov VA, Plotnikov EY, Silachev DN, Pevzner AV, Jankauskas SS, Babenko VA, Zorov SD, Balakireva AV, Juhaszova M, Sollott SJ, Zorov D (2018) Mitochondrial membrane potential. Anal Biochem 552:50-59

Publisher's Note Springer Nature remains neutral with regard to jurisdictional claims in published maps and institutional affiliations. 